\title{
Determination of glycine, glutamine, glutamate, and $\gamma$-aminobutyric acid in cerebrospinal fluids by capillary electrophoresis with light-emitting diode-induced fluorescence detection
}

\author{
Miao-Jen Lu ${ }^{\text {a }}$, Tai-Chia Chiu ${ }^{b}$, Po-Ling Chang ${ }^{a}$, \\ Hsin-Tsung $\mathrm{Ho}^{\mathrm{c}, \mathrm{d}}$, Huan-Tsung Chang ${ }^{\mathrm{a}, *}$ \\ a Department of Chemistry, National Taiwan University, Roosevelt Road, Section 4, \\ Taipei 106, Taiwan \\ ${ }^{\mathrm{b}}$ Institute of Atomic and Molecular Sciences, Academia Sinica, Taipei, Taiwan \\ ${ }^{\mathrm{c}}$ Department of Laboratory Medicine, Mackay Memorial Hospital, Taipei, Taiwan \\ ${ }^{\mathrm{d}}$ Mackay Medicine, Nursing and Management College, Taipei, Taiwan
}

Received 17 November 2004; received in revised form 14 February 2005; accepted 15 February 2005

Available online 23 March 2005

\begin{abstract}
In this article, we present a simple and cost-effective method for the determination of amino acids in cerebrospinal fluids (CSF) by using capillary electrophoresis-light-emitting diode-induced fluorescence detection. When using a deactivated capillary filled with $0.6 \%$ poly(ethylene oxide) $\left(\mathrm{PEO}, M_{\mathrm{r}} 6.0 \times 10^{5}\right)$ prepared in $10 \mathrm{mM}$ tetraborate $(\mathrm{pH} 9.3)$, we have achieved the limits of detection $(\mathrm{S} / \mathrm{N}=3)$ in the range of 10-30 nM for naphthalene-2,3-dicarboxaldehyde (NDA) derivatized amino acids (detected in the anodic side) in the absence of electroosmotic flow (EOF). In order to further improve sensitivity, stacking and separation of CSF samples in the presence of EOF has been applied. When high voltage is applied, the analytes migrating against EOF slow down and are stacked at the boundary between $2.0 \%$ PEO $\left(M_{\mathrm{r}} 8.0 \times 10^{6}\right)$ prepared in $10 \mathrm{mM}$ tetraborate $(\mathrm{pH} 9.3)$ and sample zone. The stacking approach provides the LODs at the $\mathrm{nM}$ level for the analytes (detected in the cathodic side) when injecting at $30 \mathrm{~cm}$ height for $150 \mathrm{~s}$. The two proposed methods provide comparable results for the determinations of glycine (Gly), glutamine (Gln), and glutamate (Glu) in CSF samples from patients suffered from inflammation, epilepsy, and jaundice without sample preparation, but the stacking method is more sensitive and allows for the determination of $\gamma$-aminobutyric acid (GABA).
\end{abstract}

(C) 2005 Elsevier B.V. All rights reserved.

Keywords: Amino acids; Capillary electrophoresis; Cerebrospinal fluid; Light-emitting diode-induced fluorescence detection; Stacking

\section{Introduction}

Capillary electrophoresis (CE) with laser-induced fluorescence (LIF) provides the advantages of high resolution, short analysis time, high sensitivity, and small sample size, which make it quite suitable for the routine determination of a great variety of compounds in biological samples [1-3]. Because of the brightness and spatial beam properties, lasers are com-

\footnotetext{
* Corresponding author. Tel.: +11 886223621963 ; fax: +11886223621963

E-mail address: changht@ntu.edu.tw (H.-T. Chang).
}

monly used as excitation sources. However lasers such as the $\mathrm{Ar}^{+}$and $\mathrm{He}-\mathrm{Cd}$ lasers are generally expensive, relatively bulky, and have limited lifetimes $(\sim 3000 \mathrm{~h})$. Alternatively, light-emitting diodes (LEDs) have become very attractive light sources in CE because of the advantages of a long lifetime (>10,000 h), high intensities at a variety of wavelengths (ranging from blue to red) and comparable stability when compared with those of conventional light source such as $\mathrm{Hg}$ and Xe lamps, small sizes, and ease of operation [4-6]. Because the intensities are weaker and the emission lights are broader, LED-induced fluorescence detection (LEDIF) is less sensitive for analytes than LIF detection. 
Derivatization is necessary to enhance detection signals when the analytes such as most amino acids do not possess intrinsic fluorescence. Many fluorophores have been tested to form highly fluorescent derivative compounds with amino acids [7-10]. Among these, naphthalene-2,3dicarboxaldehyde (NDA) is the most common reagent for labeling amino acids [10]. NDA reacts with primary amines in the presence of cyanide to produce cyano[ $f]$ benzoisoindole (CBI) products with high quantum yields (e.g., $\Phi_{\mathrm{f}}=0.8$ for Gly derivatives) [11]. CBI products exhibit two weaker excitation maxima in the visible region at approximately 420 and $440 \mathrm{~nm}$ and fluoresce at $490 \mathrm{~nm}$ after excitation [11].

With the advantages of LED and NDA addressed above, LEDIF detection should be sensitive enough to detect amino acids in biological samples such as blood, cerebrospinal fluid (CSF), and urine. If a greater sensitivity is required, a stacking technique in conjunction with CE-LEDIF can be applied. A number of sample stacking techniques have been demonstrated, including field amplification injection, isotachophoresis, and pH-mediated approaches [12-14]. Generally, it is much easier and straightforward for the stacking of analytes prepared in low-conductivity media, because salt could cause poor stacking efficiency and loss of resolution as a result of Joule heating $[15,16]$. However, in view of life science, techniques allowing stacking of high-salt samples are more desired. Recently, we have developed a stacking method for the analysis of proteins in urine samples (up to $0.18 \mu \mathrm{L}$ ) without any sample pretreatment [16]. To minimize salt effects, a short plug of low $\mathrm{pH}$ buffer was applied after sample injection.

CSF is secreted in the brain and is in a steady state with the fluid surrounding brain cells. It plays a critical role in providing a constant chemical environment for neurons and glia and is the body fluid most likely to reflect a disturbance of the amino acids metabolic pathway [17]. Changes in amino acids level in patients' CSFs have been found to be related to neurological and psychiatric disorders [18]. The separation of neurotransmitters in brain microdialysis samples by CE-LIF using NDA was completed in $10 \mathrm{~min}$, which provided the detection limits of $3,15,5 \mathrm{nM}$ for $\gamma$-aminobutyric acid (GABA), glutamic acid (Glu), and L-aspartate (L-Asp), respectively [19]. That study also demonstrated that applications of $0.1 \mathrm{mM}$ nipecotic acid, a GABA uptake blocker, $1 \mathrm{mM}$ pyrrolidine-2,4-dicarboxylic acid, a Glu/Asp uptake blocker, and $100 \mathrm{mg} / \mathrm{kg}$ of mercaptopropionic acid, a GABA synthesis inhibitor, induce a significant $100-200 \%$ increase, no change, and a $40 \%$ decrease ( 10 min after injection) in the concentration of GABA in striatum microdialysates, respectively. A similar technique was applied to monitoring vigabatrin, an antiepileptic drug, and amino acids neurotransmitters in microdialysates from the rat striatum during intercerebral infusion of the drug [20]. The study suggested that vigabatrin induces an increase in extracellular GABA concentration in relation with the drug concentration at the site of the biochemical changes and an unexpected increase in extracellular Glu.
In this research, we developed a sensitive and costeffective CE method for the analysis of amino acids, including, glycine (Gly), Gln and GABA using a violet-LED as the light source. In order to provide greater sensitivity for detecting GABA, a stacking technique using poly(ethylene oxide) (PEO) was applied. The usefulness of the proposed methods was tested by analyzing CSF samples from patients suffered from different diseases, including inflammation, epilepsy, and jaundice.

\section{Experimental}

\subsection{Chemicals}

All chemicals for preparing buffer solutions and amino acids were obtained from Sigma (St Louis, MO, USA), besides polymers. PEO $\left(M_{\mathrm{r}} 6.0 \times 10^{5}\right.$ and $\left.8.0 \times 10^{6}\right)$ and poly(vinyl pyrrolidone) (PVP) $\left(M_{\mathrm{r}} 1.3 \times 10^{6}\right)$ were obtained from Aldrich (Milwaukee, WI, USA). NDA was obtained from Tokyo Chemical Industry (Tokyo, Japan) and dissolved in analytical grade methanol which was obtained form J.T. Baker (Phillipsburg, NJ, USA). Sodium dihydrogen phosphate and sodium hydroxide were used to prepare low-pH phosphate buffer $(10 \mathrm{mM} ; \mathrm{pH} 5.0)$. Tris(hydroxymethyl)aminomethane (Tris) was used to prepare TB buffers ( $\mathrm{pH}$ 9.0) by adjustment with suitable amounts of boric acid. The concentration of TB buffer refers to the molarity of Tris. PEO solutions were prepared in different separation buffers (TB and tetraborate buffers) [21]. For simplicity, the polymer solution prepared from different sizes of PEO molecules is presented as $\operatorname{PEO}(X)$; for example, $0.5 \%$ $\mathrm{PEO}(8.0)$ means that the polymer solution was prepared from dissolving $0.5 \mathrm{~g}$ PEO $\left(M_{\mathrm{r}} 8.0 \times 10^{6}\right)$ in $100 \mathrm{~mL}$ of water, TB buffer, or tetraborate buffer.

\subsection{Apparatus}

The basic design of the CE-LEDIF system has been previously described [5]. Briefly, a high-voltage power supply (Gamma High Voltage Research Inc., Ormond Beach, FL, USA) was used to drive electrophoresis. The entire CE system was placed in a black box with a high-voltage interlock. For safety, the high-voltage end of the separation system was put in a laboratory-made plexiglass box. A violet LED (In$\mathrm{GaN}$ ) with a maximum output at $405 \mathrm{~nm}$ with a full-width-athalf-maximum (FWHT) of $30 \mathrm{~nm}$ was obtained from KwangHwa Electronic Material (Taichung, Taiwan) and was used for excitation. A laboratory-made power supply with adjusted voltages up to $5.0 \mathrm{~V}$ was used to drive the LED. In this study, the applied voltage of LED was set at $3.9 \mathrm{~V}$, which provided an output power of $0.04 \mathrm{~mW}$ at $405 \mathrm{~nm}$. We note that the LED was used for more than 1 year $(>3000 \mathrm{~h})$. The excitation light was focused on the capillary with a $40 \times$ objective (numerical aperture $=0.65$ ). The fluorescence was collected with a $10 \times$ objective (numerical aperture $=0.25$ ). One inter- 
ference filter ( $488 \mathrm{~nm}$ with a FWHM of $10 \mathrm{~nm}$ ) was used to block scattered lights before the emitted light reached the photomultiplier tube (R928, Hamamatsu Photonics K.K., Shizuoka-Ken, Japan). The fluorescence signal was directly transferred through a $10 \mathrm{k} \Omega$ resistor to a 24-bit A/D interface at $10 \mathrm{~Hz}$ (Borwin, JMBS Developments, Le Fontanil, France) and stored in a personal computer. Fused-silica capillary with $75 \mu \mathrm{m}$ i.d. and $365 \mu \mathrm{m}$ o.d. was purchased from Polymicro Technologies (Phoenix, AZ, USA). The capillary length is $50 \mathrm{~cm}$ ( $40 \mathrm{~cm}$ to detector) for the analysis of small injected volumes of samples (ca. $10 \mathrm{~nL}$ ), while it is $60 \mathrm{~cm}$ (50 cm to detector) when conducting stacking and separation experiments.

ADV-E viscometer (Brookfield Engineering Laboratories Inc., Middleboro, MA, USA) was employed to measure the viscosity of PEO solutions in a constant-temperature bath at $25.0 \pm 0.2^{\circ} \mathrm{C}$. All measurements were performed in triplicate.

\subsection{Sample handling and derivatization procedure}

CSF samples were taken from the bottom part of the lumbar spinal column while the patients were in a sitting position. The patients were all children who were diagnosed in the department of clinical laboratory of Mackay Memorial Hospital (Taipei, Taiwan). Derivatization procedure of amino acids with NDA in the presence of cyanide was modified from the literature [22]. The derivatization was performed in $1.5 \mathrm{~mL}$ centrifuge tubes. The standard amino acids used to investigate the performance of the separation and stacking approaches in this study are alanine (Ala), GABA, Gly, histidine (His), and phenylalanine (Phe). For standard amino acids, $1.0 \mathrm{~mL}$ reaction mixtures $(\mathrm{pH} 9.3)$ containing amino acids $(10 \mu \mathrm{M}), \mathrm{NaCN}(1.0 \mathrm{mM}), \mathrm{NDA}(1.0 \mathrm{mM})$, and sodium tetraborate $(1.0 \mathrm{mM})$, were prepared. For real samples, $50 \mu \mathrm{L}$ CSF samples without any pretreatment were mixed with $50 \mu \mathrm{L}$ aqueous solution ( $\mathrm{pH}$ 9.3) consisting of $2.0 \mathrm{mM} \mathrm{Na}_{2} \mathrm{~B}_{4} \mathrm{O}_{7}, 2.0 \mathrm{mM} \mathrm{NaCN}$, and $2.0 \mathrm{mM}$ NDA. To determine the concentrations of GABA, Gln, Glu, and Gly in CSF, $1-3 \mu \mathrm{L}$ of standards were spiked to $100 \mu \mathrm{L}$ of mixtures containing CSF samples $(50 \mu \mathrm{L})$ prior to analysis. The solutions were allowed to react for $20 \mathrm{~min}$ in room temperature before injection into the capillary.

\subsection{Separation in the absence of EOF}

The capillary used was dynamically coated with $5.0 \%$ PVP prepared in water at ambient temperature overnight. Before coating the second layer of the capillary wall with $0.5 \%$ $\operatorname{PEO}(8.0)$ prepared in water at room temperature for $2 \mathrm{~h}$, the polymer solution inside the capillary was flushed out with water [23]. The capillary was filled with $0.6 \% \mathrm{PEO}(0.6)$ solution (prepared in tetraborate buffer; condition A) prior to separation. Electrokinetic injection was conducted at $-10 \mathrm{kV}$ for $10 \mathrm{~s}$. Separation was carried out at $-10 \mathrm{kV}$ using $40 \mathrm{~cm}$ capillaries (30 cm in effective length). After each run, the cap- illary was recoated with PEO for 2 min to achieve optimum resolution and reproducibility.

\subsection{Separation in the presence of $E O F$}

Prior to analysis, capillaries were treated with $0.5 \mathrm{M}$ $\mathrm{NaOH}$ overnight [24]. After each run, capillaries were flushed with $0.5 \mathrm{M} \mathrm{NaOH}$ at $1 \mathrm{kV}$ for $10 \mathrm{~min}$ to remove PEO solutions and to refresh the capillary wall, and subsequently filled with TB or tetraborate buffers. This treatment was quite successful to regenerate high and reproducible EOF (RSD $<1.5 \%)$. The capillaries are $40 \mathrm{~cm}$ in total length and $30 \mathrm{~cm}$ in effective length. During separation, PEO solution prepared in TB buffer (condition B) or in tetraborate buffer (condition C) was introduced to the capillary by EOF from the anodic side. Hydrodynamic injection was applied at $30 \mathrm{~cm}$ height for a time over a range from 10 to $180 \mathrm{~s}$. After samples injection, a low $\mathrm{pH}$ plug of phosphate buffer $(10 \mathrm{mM}, \mathrm{pH} 5.0)$ was applied [16]. The separation was conducted at $15 \mathrm{kV}$.

\section{Results and discussion}

\subsection{Separations in the presence and absence of EOF}

In terms of the fluorescence quantum yield and stability of the amino acid derivatives, separation conducted at $\mathrm{pH} 9.5$ is preferred [25]. From the view point of the use of $\mathrm{PEO}, \mathrm{pH}$ greater than 10.0 is not suitable because of accelerated hydrolysis of PEO. In our previous study, we demonstrated that reproducibility is poor at $\mathrm{pH}<8.0$ as a result of serious $\mathrm{PEO}$ adsorption on the capillary wall [26]. For these reasons, we only investigated the effect of PEO solution and background electrolytes on the separation of amino acid derivatives at $\mathrm{pH} 9.0$ and 9.3. It has been suggested that $\operatorname{PEO}(0.6)$ provides better resolution for proteins than does $\operatorname{PEO}(8.0)$ [27]. However, we did not see a similar result for separating amino acid derivatives, mainly because the separation of small analytes is not according to the sieving mechanism. According to literatures $[28,29]$, we suggested that PEO molecules interact with the amino acid derivatives through hydrogen bonding between polyethylene oxide chains of polymer and carboxylic groups or hydroxyl groups of the amino acid derivatives. In this study, we separately evaluated the separations of amino acid derivatives in the presence and absence of EOF using PEO. In the absence of EOF, low-viscosity $\operatorname{PEO}(0.6)$ solution (0.6\%) was used for the sake of ease of filling a capillary and speed (the electrophoretic mobility decreases with increasing viscosity). In the presence of EOF, only high-concentration PEO (8.0) solutions $(\geq 1.5 \%)$ were tested because the stacking efficiency and resolution increase with increasing the viscosity [30,31]. Using $\operatorname{PEO}(8.0)$ at the concentration less than $1.5 \%$, the loss of resolution is problematic, mainly due to high bulk EOF and poor stacking.

As shown in Table 1, the separation conducted in the absence of EOF using $0.6 \% \mathrm{PEO}(0.6)$ provides longer migra- 
Table 1

Comparison of three different conditions for the analyses of five model amino acids with respect to migration time, peak width, and LOD

\begin{tabular}{|c|c|c|c|c|c|c|c|c|c|}
\hline & \multicolumn{3}{|c|}{$\mathrm{A}^{\mathrm{a}}(0.6 \% \operatorname{PEO}(0.6))$} & \multicolumn{3}{|c|}{$\mathrm{B}^{\mathrm{b}}(1.5 \% \operatorname{PEO}(8.0))$} & \multicolumn{3}{|c|}{$\mathrm{C}^{\mathrm{b}}(2.0 \% \operatorname{PEO}(8.0))$} \\
\hline & Time (min) & $\begin{array}{l}\text { Peak width } \\
(\min )\end{array}$ & $\operatorname{LOD}^{\mathrm{c}}(\mathrm{nM})$ & Time (min) & $\begin{array}{l}\text { Peak width } \\
(\min )\end{array}$ & LOD $(\mathrm{nM})$ & Time (min) & $\begin{array}{l}\text { Peak width } \\
(\min )\end{array}$ & LOD $(\mathrm{nM})$ \\
\hline Gly & 10.6 & 0.32 & 9.3 & 10.25 & 0.05 & 50.6 & 8.71 & 0.09 & 44.2 \\
\hline Ala & 11.2 & 0.44 & 22.1 & 9.71 & 0.05 & 84.1 & 8.07 & 0.10 & 75.4 \\
\hline GABA & 12.6 & 0.37 & 14.2 & 9.23 & 0.04 & 48.9 & 7.33 & 0.07 & 44.7 \\
\hline His & 14.4 & 0.38 & 27.5 & 8.74 & 0.04 & 85.2 & 6.67 & 0.09 & 76.9 \\
\hline Phe & 16.1 & 0.46 & 19.4 & 8.44 & 0.03 & 48.7 & 6.24 & 0.09 & 40.9 \\
\hline
\end{tabular}

${ }^{a}$ Capillary, effective (total) length $40 \mathrm{~cm}(30 \mathrm{~cm})$; separation voltage, $-10 \mathrm{kV}$; in the absence of EOF; PEO prepared in $10 \mathrm{mM}$ borate buffer (pH 9.3 ).

b Capillary, effective (total) length $40 \mathrm{~cm}(30 \mathrm{~cm})$; separation voltage, $15 \mathrm{kV}$; in the presence of EOF; PEO prepared in $0.2 \mathrm{M}$ TB buffer (pH 9.0$)$ and $10 \mathrm{mM}$ borate buffer ( $\mathrm{pH} 9.3)$ in conditions $\mathrm{B}$ and $\mathrm{C}$, respectively.

c The concentrations of amino acids used for measuring migration times and peak widths are $10^{-6} \mathrm{M}$. The linear regression coefficients $\left(R^{2}\right)$ for the peak height and peak area against the analyte concentration at the range of $10^{-5}$ to $10^{-7} \mathrm{M}$ are both $\geq 0.98$. The LODs at the signal-to-noise ratio (S/N) 3 were calculated from the electropherograms when injecting $10^{-7} \mathrm{M}$ analytes.

tion times when compared to those in the presence of EOF. Broader peak profiles are another drawback when the separation was conducted in the absence of EOF. Band broadening is mainly due to greater diffusion at lower-viscosity media and longer separation times. Despite these shortages, this condition provides better resolution and stable baseline. In the presence of EOF, the analytes migrated against EOF and were detected in the cathodic side. We note that the migration order is reversed (detection point: anodic side) to that in the absence of EOF. We found that the optimum $\operatorname{PEO}(8.0)$ concentrations in terms of speed and efficiency were 1.5 and $2.0 \%$ when they were prepared in $200 \mathrm{mM} \mathrm{TB}(\mathrm{pH} 9.0)$ and $10 \mathrm{mM}$ tetraborate buffers ( $\mathrm{pH} 9.3$; containing sodium ions), respectively. It is worthy noting that the five amino acids were well resolved $\left(R_{\mathrm{S}}>1.5\right)$. Table 1 also shows that the separation is faster in the latter case as a result of a greater EOF at high ionic strengths [30]. When using 1.5\% $\mathrm{PEO}(8.0)$ solution prepared in $200 \mathrm{mM}$ TB $(\mathrm{pH} 9.0)$ and $2.0 \% \mathrm{PEO}(8.0)$ prepared in $10 \mathrm{mM}$ tetraborate buffers ( $\mathrm{pH} 9.3$ ), the EOF mobility values are $1.98 \times 10^{-4}$ and $5.03 \times 10^{-4} \mathrm{~cm}^{2} \mathrm{~V}^{-1} \mathrm{~s}^{-1}$, respectively. On the basis of the fact that the viscosity values of 1.5 and $2.0 \%$ PEO are 1642 and $4118 \mathrm{cP}$, respectively, we suggest that a greater EOF in $10 \mathrm{mM}$ borate buffer ( $\mathrm{pH} 9.3$ ) is due to reduced PEO adsorption on the capillary wall at a high ionic strength. We note that the sensitivity is better in the absence of EOF as a result of a stable baseline and lower fluorescence background. For example, the limit of detections (LODs) at $\mathrm{S} / \mathrm{N}=3$ are 9.3 and $44.2 \mathrm{nM}$ for Gly and 14.2 and $44.7 \mathrm{nM}$ for GABA when using conditions $\mathrm{A}$ and $\mathrm{C}$ listed in Table 1 , respectively.

\subsection{Stacking and separation}

It is extremely important to detect trace amounts of amino acids and peptides in biological samples. In order to further improve the sensitivity, we applied a stacking technique on the basis of viscosity changes [24,30-32]. It is worthy noting again that stacking efficiency is greater when using highviscosity PEO solution. However, filling is a problem. On the basis of our experience, the maximum sample injection length is also shorter when using a capillary filled with PEO solution (in the absence of EOF) than that filled with TB buffer while achieving similar resolving powers. Thus, only stacking and separation of amino acid derivatives in the presence of EOF using 2.0\% PEO(8.0) was tested. The amino acid derivatives (anions) migrating against EOF slow down and are stacked at the boundary between sample zone and PEO (neutral) solutions as a result of increases in viscosity and possible interactions with PEO molecules (hydrogen bonding). As shown in Fig. 1, five peaks were well separated using 2.0\% PEO(8.0) when the sample was injected at $30 \mathrm{~cm}$ height for $150 \mathrm{~s}$ (about $0.33 \mu \mathrm{L}$ ). We note that the migration orders are different in the presence and absence of EOF. A longer separation time (versus condition $\mathrm{C}$ in Table 1) is mainly due to a small EOF mobility because $\mathrm{PEO}$ adsorption is significant when injecting a long plug of sample prepared

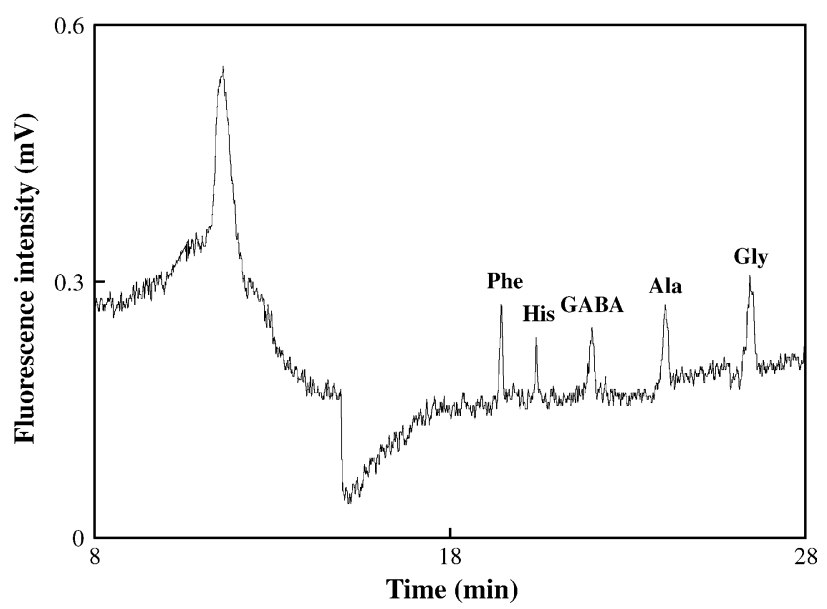

Fig. 1. Electropherogram of stacking and separation of five amino acids $(50 \mathrm{nM})$ in the presence of EOF. Capillary is $60 \mathrm{~cm}$ in total length and $50 \mathrm{~cm}$ in effective length. Prior to sample injection, the capillary was filled with $10 \mathrm{mM}$ tetraborate buffer ( $\mathrm{pH} 9.3$ ). Hydrodynamic injection was performed at $30 \mathrm{~cm}$ height for $150 \mathrm{~s}$ and separation was conducted at $15 \mathrm{kV}$ using $2.0 \%$ $\mathrm{PEO}(8.0)$ prepared in $10 \mathrm{mM}$ tetraborate buffer ( $\mathrm{pH} 9.3)$. 
in a low ionic strength [31]. The plate numbers for the five analytes are all greater than $0.45 \mathrm{million} / \mathrm{m}$, indicating good stacking. The LODs $(\mathrm{S} / \mathrm{N}=3)$ for Phe, His, GABA, Ala, and Gly are 3.4, 6.8, 4.5, 7.0, and $4.1 \mathrm{nM}$, respectively. When compared to the results shown in Table 1 (condition C), the sensitivity improvements are over 10-fold for all the analytes. We point out that the linear regression coefficients for the peak height (or area) against the analyte concentration for all model analytes over the injection range $10-150 \mathrm{~s}$ are higher than 0.98 .

\subsection{Analysis of CSF in the absence of EOF}

As addressed above, analysis of amino acid derivatives in the absence of EOF is sensitive (at the $\mathrm{nM}$ level), which shows the potential of this method for analyzing CSF samples. Fig. 2A presents the separation of a CSF sample from a patient suffered from inflammation, exhibiting several identified peaks corresponding to Glu, Gly, Gln, serine (Ser), valine (Val), isoleucine (Ile), and Phe. Fig. 2B-D further show the separations of CSF samples from patients suffered from epilepsy, jaundice, as well as jaundice and epilepsy. The reproducibility of this method is good; RSD for the migration times less than $2.5 \%$ for the same sample. We point out that the fluorescence of the amino acid derivatives is quenched about 10 times by salts. The migration times for Glu, Gly, and Gln are different for various CSF samples, resulting from matrix effects. It is thus important to apply a standard addition method to minimize the matrix interference. In this study, suitable amounts ( 1 to $80 \mu \mathrm{M}$ ) of corresponding standard analytes were spiked to the CSF samples. The peak heights as a function of the spiked analyte concentrations are linear for Glu, Gly, and Gln. The linear regression coefficients for Glu, Gly, and Gln in the eight CSF samples are all greater than $0.97,0.93$, and 0.96 , respectively. The concentrations of Glu, Gly, and Gln in different CSF samples are summarized in Table 2. The concentrations of Glu in the CSF samples are all in the normal range, while some of those for Gly and Gln are different from the normal values [33].

\subsection{Stacking of CSF}

Owing to matrix (salt) effect and poor sensitivity, CE in the presence of EOF might not be suitable for CSF samples (high salt and proteins). Our hypothesis is supported by the electropherogram depicted in Fig. 3A, showing that only several broad peaks were detected when injected the CSF sample ( $30 \mathrm{~cm}$ height for $10 \mathrm{~s})$. To overcome the problem, we injected a low-pH plug $(\mathrm{pH} 5.0 ; 10 \mathrm{~s})$ after sample injection $(30 \mathrm{~cm}$ height for $180 \mathrm{~s}$ ) [16]. The low-pH plug provides at least two benefits for the analysis: (1) the effect of anions such as $\mathrm{Cl}^{-}$, $\mathrm{H}_{2} \mathrm{PO}_{4}{ }^{-}$, and $\mathrm{HPO}_{4}{ }^{2-}$ on stacking is minimized; and (2) the analytes slow down as a result of $\mathrm{pH}$ changes, thereby increasing stacking efficiency. Fig. 3B shows better sensitivity and thus more peaks were detected, including GABA that
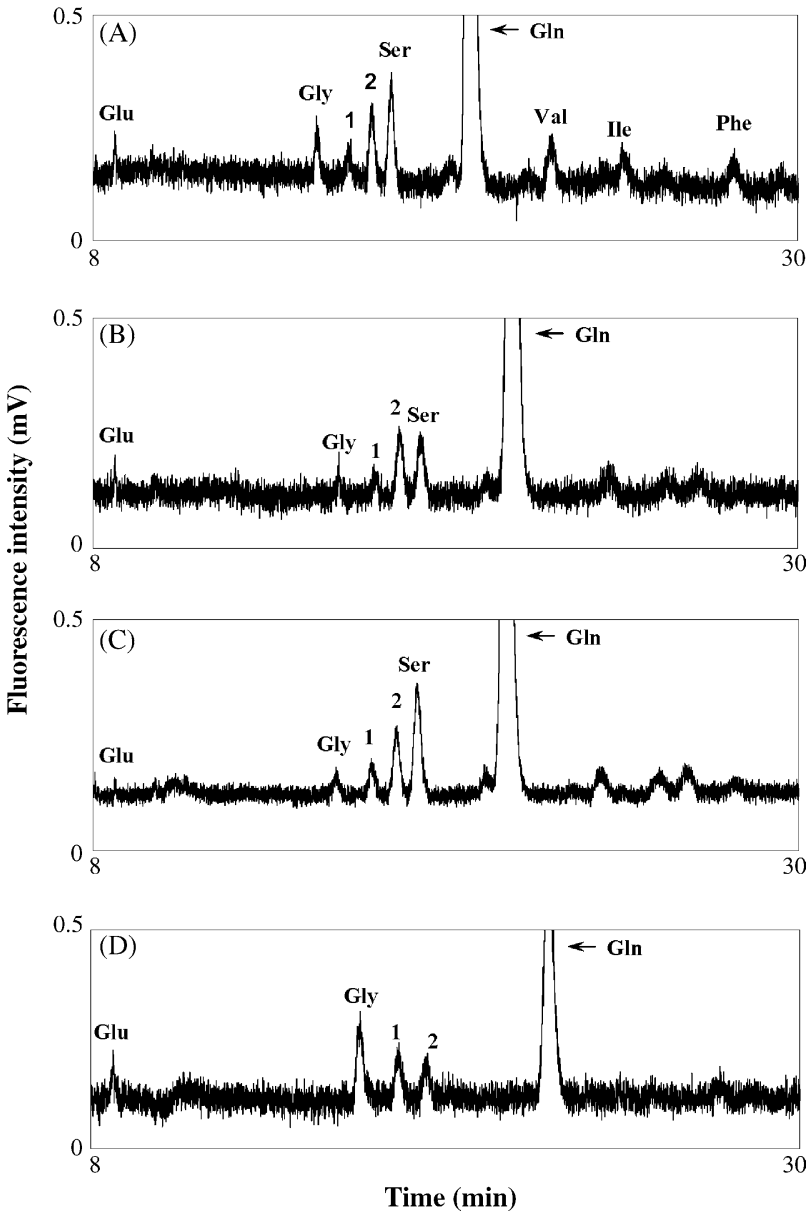

Fig. 2. Separations of amino acid derivatives in different CSF samples in the absence of EOF using $0.6 \% \mathrm{PEO}(0.6)$ prepared in $10 \mathrm{mM}$ tetraborate $(\mathrm{pH}$ 9.3). CSF samples are from patients suffered from diseases of: (A) inflammation; (B) epilepsy; (C) jaundice; (D) epilepsy and jaundice. Capillary is $40 \mathrm{~cm}$ in total length and $30 \mathrm{~cm}$ in effective length. Electrokinetic injection was conducted at $-10 \mathrm{kV}$ for $10 \mathrm{~s}$ and separation was carried out at $-10 \mathrm{kV}$. The capillary was coated with $5.0 \% \mathrm{PVP}(1.3)$ and then with $0.5 \% \mathrm{PEO}(8.0)$. Peaks assigned as numbers 1 and 2 in the electropherograms separately correspond to two different unidentified analytes.

was not detected using the method in the absence of EOF. We note that the reason for not detecting GABA in CSF samples in the absence of EOF is because the method is not sensitive enough to detect $10^{-7}$ to $10^{-8} \mathrm{M} \mathrm{GABA}$, which is its normal concentration range in CSF [34]. Gly and Glu possessing greater electrophoretic mobilities against EOF, they were detected after $25.0 \mathrm{~min}$ (not shown in the electropherograms). Fig. 4 shows the electropherograms for the analyses of CSF samples from patients suffered from inflammation, epilepsy, jaundice, and epilepsy coupled with jaundice. The reproducibility of this method is good; RSD for the migration times less than $2.5 \%$. In order to quantify the concentrations of Gln and GABA, the standards at the concentration ranges of $100-800 \mu \mathrm{M}$ and $20-200 \mathrm{nM}$ were spiked to the samples, respectively. The peak heights as a function of the spiked analyte concentrations are linear with the linear regression 
Table 2

Quantification of amino acids in CSF from patients suffered from inflammation, epilepsy and jaundice in the absence of EOF

\begin{tabular}{|c|c|c|c|c|c|c|c|}
\hline \multirow[t]{2}{*}{ Syndrome/disease } & \multirow[t]{2}{*}{ Patients } & \multicolumn{3}{|c|}{ Migration time (min) } & \multicolumn{3}{|c|}{ Concentration $(\mu \mathrm{M})^{\mathrm{a}}$} \\
\hline & & Glu & Gly & Gln & Glu & Gly & Gln \\
\hline Inflammation & 1 & $8.7 \pm 0.1^{\mathrm{b}}$ & $14.9 \pm 0.2$ & $19.7 \pm 0.3$ & $4.9 \pm 0.5(0.98)^{\mathrm{c}}$ & $16 \pm 1(0.94)$ & $610 \pm 20(0.97)$ \\
\hline Inflammation & 2 & $8.6 \pm 0.1$ & $14.7 \pm 0.1$ & $19.5 \pm 0.2$ & $4.4 \pm 0.1(0.98)$ & $14 \pm 1(0.95)$ & $490 \pm 18(0.96)$ \\
\hline Inflammation & 3 & N.D. ${ }^{d}$ & $14.8 \pm 0.2$ & $19.8 \pm 0.3$ & N.D. ${ }^{c}$ & $12 \pm 4(0.93)$ & $130 \pm 12(0.98)$ \\
\hline Inflammation & 4 & $8.5 \pm 0.1$ & $14.5 \pm 0.2$ & $19.3 \pm 0.3$ & $4.1 \pm 0.6(0.97)$ & $10 \pm 2(0.93)$ & $420 \pm 18(0.96)$ \\
\hline Epilepsy & 5 & $8.7 \pm 0.1$ & $15.7 \pm 0.2$ & $21.1 \pm 0.4$ & $4.8 \pm 0.9(0.97)$ & $12 \pm 1(0.95)$ & $310 \pm 16(0.96)$ \\
\hline Jaundice & 6 & $8.6 \pm 0.1$ & $15.6 \pm 0.2$ & $20.9 \pm 0.3$ & $3.2 \pm 0.1(0.97)$ & $12 \pm 1(0.95)$ & $460 \pm 17(0.97)$ \\
\hline Jaundice & 7 & $8.7 \pm 0.1$ & $15.4 \pm 0.2$ & $20.5 \pm 0.3$ & $5.2 \pm 0.7(0.98)$ & $8 \pm 2(0.93)$ & $120 \pm 10(0.97)$ \\
\hline Epilepsy and jaundice & 8 & $8.7 \pm 0.2$ & $16.4 \pm 0.3$ & $22.3 \pm 0.4$ & $5.1 \pm 1.2(0.97)$ & $25 \pm 5(0.93)$ & $260 \pm 13(0.96)$ \\
\hline Normal range $\mathrm{e}^{\mathrm{e}}$ & & & & & $1-48$ & $3-11$ & $335-885$ \\
\hline
\end{tabular}

${ }^{\text {a }}$ Capillary was filled with $0.6 \% \mathrm{PEO}(0.6)$ prepared in $10 \mathrm{mM}$ tetraborate buffer $(\mathrm{pH} 9.3)$; the separations were conducted at $-10 \mathrm{kV}$; the electropherograms for samples from patients 1, 5, 6, and 8 are depicted in Fig. 2; other conditions are the same as method A in Table 1.

${ }^{\mathrm{b}}$ Data of quantitative variables are expressed as mean \pm S.D. $(n=5)$.

${ }^{c}$ Linear regression coefficients.

d Not detected.

e Data from Ref. [33].

coefficients for Gln and GABA in the eight CSF samples are greater than 0.97 and 0.93 , respectively. The migration times and concentrations of Gln and GABA in the CSF samples are summarized in Table 3. We note that the GABA concentrations in the CSF samples from patients suffered from

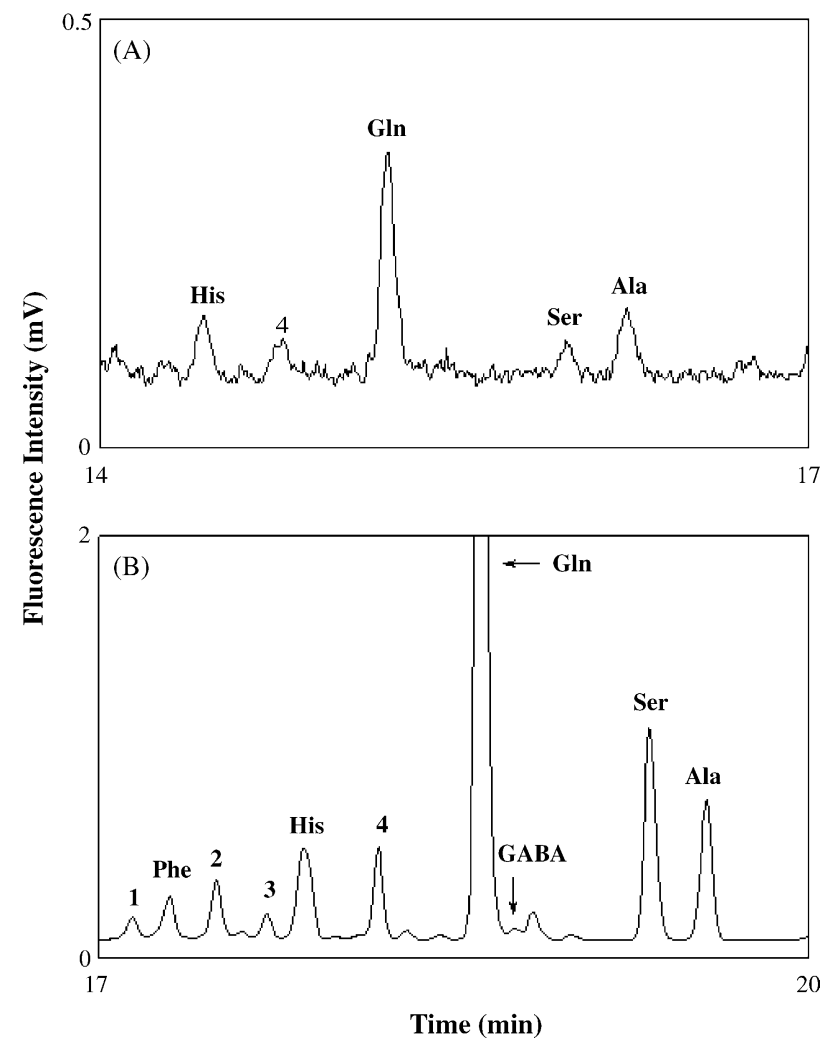

Fig. 3. Electropherograms of amino acid derivatives in a CSF sample in the presence of EOF using 2.0\% PEO(8.0): (A) the sample (as Fig. 2A) was injected hydrodynamically at $30 \mathrm{~cm}$ height for $10 \mathrm{~s}$; (B) hydrodynamic injection was conducted at $30 \mathrm{~cm}$ height for $180 \mathrm{~s}$. After sample injection, a low-pH (pH 5.0) plug was applied at $30 \mathrm{~cm}$ height for $10 \mathrm{~s}$. Other conditions are the same as in Fig. 1. Peaks assigned as numbers 1-4 separately correspond to four different unidentified analytes. inflammation and epilepsy are in the range of $40-120 \mathrm{nM}$, which is higher than the normal range. This is probably induced by drugs (such as vigabatrin) that patients took. It has been reported that antiepileptic drugs increase the concentration of GABA in CSF of patients [20]. The concentrations
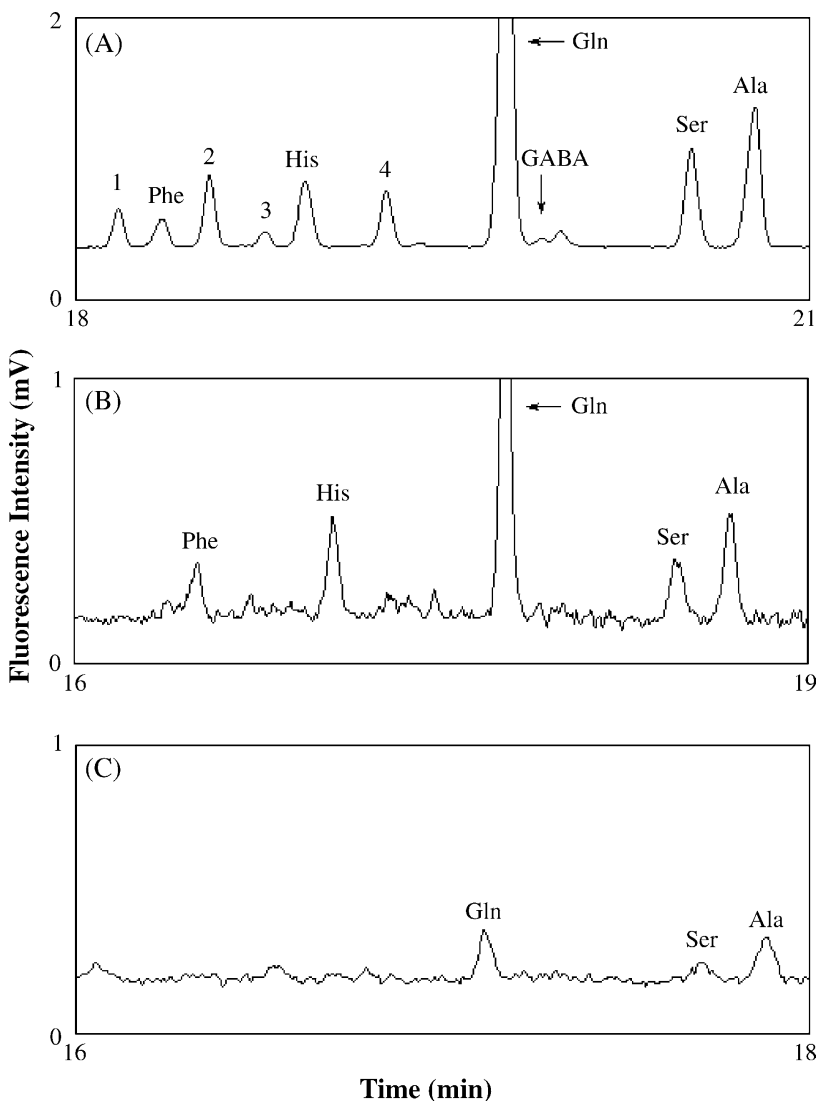

Fig. 4. Separations of amino acid derivatives in different CSF samples in the presence of EOF using 2.0\% $\mathrm{PEO}(8.0)$. CSF samples are from patients suffered from diseases of: (A) epilepsy; (B) jaundice; (C) epilepsy and jaundice. Other conditions are the same as in Fig. 3B. 
Table 3

Quantification of amino acids in CSF from patients suffered from inflammation, epilepsy and jaundice in the presence of EOF

\begin{tabular}{|c|c|c|c|c|c|}
\hline \multirow[t]{2}{*}{ Syndrome/disease } & \multirow[t]{2}{*}{ Patients } & \multicolumn{2}{|c|}{ Migration time $(\min )^{\mathrm{a}}$} & \multicolumn{2}{|c|}{ Concentration $(\mu \mathrm{M})^{\mathrm{a}}$} \\
\hline & & Gln & GABA & $\overline{\mathrm{Gln}}(\mu \mathrm{M})$ & GABA (nM) \\
\hline Inflammation & 1 & $18.6 \pm 0.3^{b}$ & $18.8 \pm 0.4$ & $640 \pm 20(0.98)^{c}$ & $110 \pm 8(0.94)$ \\
\hline Inflammation & 2 & $18.0 \pm 0.2$ & $18.3 \pm 0.3$ & $480 \pm 18(0.97)$ & $90 \pm 7(0.95)$ \\
\hline Inflammation & 3 & $18.4 \pm 0.1$ & $18.6 \pm 0.1$ & $140 \pm 10(0.98)$ & $50 \pm 4(0.93)$ \\
\hline Inflammation & 4 & $18.3 \pm 0.3$ & $18.5 \pm 0.2$ & $430 \pm 16(0.98)$ & $80 \pm 6(0.96)$ \\
\hline Epilepsy & 5 & $19.8 \pm 0.3$ & $20.0 \pm 0.4$ & $320 \pm 17(0.97)$ & $50 \pm 3(0.93)$ \\
\hline Jaundice & 6 & $18.0 \pm 0.2$ & N.D. ${ }^{d}$ & $440 \pm 14(0.98)$ & N.D. \\
\hline Jaundice & 7 & $18.9 \pm 0.3$ & N.D. & $120 \pm 13(0.97)$ & N.D. \\
\hline Epilepsy and jaundice & 8 & $17.1 \pm 0.4$ & N.D. & $270 \pm 14(0.97)$ & N.D. \\
\hline Normal range & & & & $335-885^{\mathrm{e}}$ & $30-85^{\mathrm{f}}$ \\
\hline
\end{tabular}

a The electropherograms for samples from patients 1, 5, 6, and 8 are depicted in Fig. 4; experimental conditions were the same as in Fig. 3B.

b Data of quantitative variables are expressed as mean \pm S.D. $(n=5)$.

c Linear regression coefficients.

d Not detected.

e Data from Ref. [33].

${ }^{f}$ Data from Ref. [34].

of Gln in different CSF samples obtained in the presence of EOF agree with those from the method in the absence of EOF.

\section{Conclusions}

CE-LEDIF was developed for the analysis of amino acid derivatives in CSF samples in the presence or absence of EOF. When compared to CE-LIF, the system is more compact and cheaper, but provides lower sensitivity (about 10 times lower). Without applying stacking, we achieved better sensitivity but longer separation times than do the analysis of amino acid derivatives in the absence of EOF. This method has been tested for the analysis of different CSF samples from patients suffered from inflammation, jaundice, and epilepsy. Stacking and separation of amino acid derivatives in the presence of EOF provides sensitivity improvement more than 10 -fold. By applying a short plug of low-pH solution, the analysis of large volumes of CSF samples without sample pretreatment has been demonstrated. This approach allows detection of GABA (less than $1.0 \mu \mathrm{M}$ ) in CSF samples from patients suffered from inflammation and epilepsy. The results shown in this study indicate great potential of this proposed method for determining amino acids in biological samples.

\section{Acknowledgements}

This work was supported by the National Science Council of Taiwan under contract numbers NSC 93-2113-M-002-034 and 93-2113-M-002-035. T.-C. Chiu is grateful to Academia Sinica for his postdoctoral fellowship at IAMS.

\section{References}

[1] Y.-W. Lin, T.-C. Chiu, H.-T. Chang, J. Chromatogr. B 793 (2003) 37.
[2] Y. Ma, G. Liu, M. Du, I. Stayton, Electrophoresis 25 (2004) 1473.

[3] W.W.C. Quigley, N.J. Dovichi, Anal. Chem. 76 (2004) 4645.

[4] M. King, B. Paull, P.R. Haddad, M. Macka, Analyst 127 (2002) 1564.

[5] S.-J. Chen, M.-J. Chen, H.-T. Chang, J. Chromatogr. A 1017 (2003) 215.

[6] C.-H. Tsai, H.-M. Huang, C.-H. Lin, Electrophoresis 24 (2003) 3083.

[7] R.T. Kennedy, J.E. Thompson, T.W. Vickroy, J. Neurosci. Meth. 114 (2002) 39.

[8] M. Ummadi, B.C. Weimer, J. Chromatogr. A 964 (2002) 243.

[9] W.J.M. Underberg, J.C.M. Waterval, Electrophoresis 23 (2002) 3922.

[10] V. Poinsot, C. Bayle, F. Couderc, Electrophoresis 24 (2003) 4047.

[11] B.K. Matuszewski, R.S. Givens, K. Srinivasachar, R.G. Carlson, T. Higuchi, Anal. Chem. 59 (1987) 1102.

[12] D.M. Osbourn, D.J. Weiss, C.E. Lunte, Electrophoresis 21 (2000) 2768.

[13] H.-T. Chang, T.-C. Chiu, G.I.T. Lab. J. 2 (2003) 64.

[14] M. Urbanek, L. Křivánková, P. Boček, Electrophoresis 24 (2003) 466.

[15] A.R. Timerbaev, K. Fukushi, T. Miyado, N. Ishio, K. Saito, S. Motomizu, J. Chromatogr. A 888 (2000) 309.

[16] W.-L. Tseng, H.-T. Chang, J. Chromatogr. A 924 (2001) 93.

[17] B.B. Ruzicka, K.H. Jhamandas, Prog. Neurobiol. 40 (1993) 223.

[18] A.J. Shah, F. Crespi, C. Heidbreder, J. Chromatogr. B 781 (2002) 151.

[19] V. Sauvient, S. Parrot, N. Benturquia, E. Bravo-Moraton, B. Renaud, L. Denoroy, Electrophoresis 24 (2003) 3187.

[20] N. Benturquia, S. Parrot, V. Sauvinet, B. Renaud, L. Denoroy, J. Chromatogr. B 806 (2004) 237.

[21] I.-T. Kuo, T.-C. Chiu, H.-T. Chang, Electrophoresis 24 (2003) 3339.

[22] M.C. Roach, M.D. Harmony, Anal. Chem. 59 (1987) 411.

[23] S.-J. Wang, W.-L. Tseng, Y.-W. Lin, H.-T. Chang, J. Chromatogr. A 979 (2002) 261.

[24] T.-C. Chiu, Y.-W. Lin, C.-C. Huang, A. Chrambach, H.-T. Chang, Electrophoresis 24 (2003) 1730.

[25] P. de Montigny, J.F. Stobaugh, R.S. Givens, R.G. Carlson, K. Srinivasachar, L.A. Sternson, T. Higuchi, Anal. Chem. 59 (1987) 1096.

[26] W.-L. Tseng, H.-T. Chang, Electrophoresis 22 (2001) 763.

[27] Y. He, E.S. Yeung, J. Proteome Res. 1 (2002) 273.

[28] Y. Esake, Y. Yamaguchi, K. Kano, M. Goto, Anal. Chem. 66 (1994) 2441.

[29] M.-M. Hsieh, C.-E. Hsu, W.-L. Tseng, H.-T. Chang, Electrophoresis 23 (2002) 1633. 
[30] W.-L. Tseng, H.-T. Chang, Anal. Chem. 72 (2000) 4805.

[31] W.-L. Tseng, M.-M. Hsieh, S.-J. Wang, H.-T. Chang, J. Chromatogr. A 894 (2000) 219.

[32] P.-L. Chang, I.-T. Kuo, T.-C. Chiu, H.-T. Chang, Anal. Bioanal. Chem. 379 (2004) 404
[33] G. Nouadje, H. Rubie, E. Chatelut, P. Canal, M. Nertz, Ph. Puig, F. Couderc, J. Chromatogr. A 717 (1995) 293.

[34] T. Goto, N. Matsuo, T. Takashi, Brain Develop. 23 (2001) 24. 